\author{
Oriol Aspachs* \\ Ruben Durante* \\ Alberto Graziano* \\ Josep Mestres* \\ Jose G. Montalvo ${ }^{* * *}$ \\ Marta Reynal-Querol ${ }^{* *}$
}

\title{
SEGUIMIENTO DE LA DESIGUALDAD EN TIEMPO REAL EN ESPAÑA DURANTE LA CRISIS DE LA COVID-19
}

Este artículo analiza la evolución de la desigualdad de ingresos en España durante la crisis de la COVID-19 en alta frecuencia. Para ello, utilizamos datos anonimizados de registros bancarios de más de tres millones de titulares de cuentas bancarias entre febrero de 2020 y julio de 2021. Los resultados muestran el fuerte aumento de la desigualdad experimentado en los primeros meses de la COVID-19, así como la recuperación posterior a medida que la economía se ha reactivado. Asimismo, analizamos el crucial papel que han jugado las ayudas públicas para mitigar el impacto de la crisis.

\section{Real-Time Inequality in Spain during the COVID-19 crisis}

This article analyzes the evolution of income inequality in Spain during the COVID-19 crisis at high frequency. To do this, we used anonymized bank data from more than three million bank account holders between February 2020 and July 2021. The results show the sharp increase in inequality experienced in the first months of COVID-19, as well as the subsequent recovery as the economy reactivated. We also analyze the key role that the public sector has played in mitigating the impact of the crisis.

Palabras clave: desigualdad, COVID-19, datos administrativos, alta frecuencia.

Keywords: inequality, COVID-19, administrative data, high frequency.

JEL: D63, E24, J31.

\footnotetext{
${ }^{*}$ CaixaBank Research.

Contacto: research@caixabank.com

** ICREA-UPF, IPEG, Barcelona School of Economics, and CEPR.

*** UPF, IPEG and Barcelona School of Economics.

Los autores agradecen el apoyo del proyecto SR20-00608 de

la Social Research Call 2020 de la Fundación "la Caixa".

Versión de noviembre de 2021.

https://doi.org/10.32796/ice.2021.923.7321
} 


\section{Introducción}

Este artículo analiza el impacto de la crisis económica generada por la COVID-19 en la desigualdad salarial. La utilización de datos masivos en tiempo real es la clave, puesto que nos permiten analizar también el papel que han jugado las ayudas públicas para mitigar el impacto de la crisis, tanto para el conjunto de la población, como en los diferentes colectivos. Al tratarse de datos en tiempo real, podemos realizar asimismo un diagnóstico de la situación actual, un elemento capital para valorar, entre otras cosas, a qué velocidad se deben ir retirando las ayudas llevadas a cabo durante los últimos trimestres.

Hasta ahora esto no era posible, puesto que normalmente hay que esperar unos años para disponer de datos sobre la distribución de los ingresos de la población. En España, por ejemplo, los microdatos de la Encuesta de Estructura Salarial (EES) disponibles en estos momentos corresponden al año 2018, y los próximos microdatos correspondientes a un año pospandemia (2022) se publicarán en el 2024. Retrasos similares ocurren en el resto de los países desarrollados.

Pero, un equipo de investigadores de la Universidad Pompeu Fabra, el Institute of Political Economy and Governance (IPEG) y CaixaBank Research hemos llevado a cabo un proyecto pionero a nivel mundial, utilizando datos bancarios (totalmente anonimizados) representativos del conjunto de la población, para construir métricas que nos permiten hacer un seguimiento de la desigualdad salarial en tiempo real, sin necesidad de esperar meses, o años. La utilización de los datos bancarios también permite identificar las transferencias que realiza el sector público, y su impacto en la distribución de ingresos en tiempo real. Concretamente, nuestro estudio analiza la evolución de la desigualdad salarial a partir de la distribución de los ingresos salariales de más de tres millones de nóminas en España. El periodo analizado comienza en 2018 hasta la actualidad.

Otras iniciativas han utilizado técnicas de big data para estudiar los impactos de la pandemia en tiempo real. Uno de los proyectos más ambiciosos a nivel internacional es el Opportunity Insights de Chetty et al. (2020), que han desarrollado un indicador de seguimiento de la economía estadounidense en tiempo real a partir de datos de tarjetas de crédito, empresas de servicios financieros, etc. Otros ejemplos son Cicala (2020), que utiliza datos sobre la electricidad para medir cambios en la actividad industrial en Europa y Bick y Blandin (2021), que realizan una Encuesta de Población Activa en tiempo real para construir estimaciones de alta frecuencia de empleo, horas trabajadas e ingresos en EE UU. Nuestro trabajo, sin embargo, es pionero en utilizar datos bancarios para construir un indicador de alta frecuencia de la desigualdad salarial, sin las limitaciones de los datos administrativos o de las medidas de alta frecuencia basadas en encuestas. A continuación, hacemos un análisis de los principales resultados obtenidos hasta el día de hoy.

\section{Construcción de los datos}

Para llevar a cabo el estudio, analizamos los datos de las nóminas depositadas en CaixaBank, debidamente anonimizadas y aplicando técnicas de big data para gestionar el gran volumen de información disponible (analizamos la evolución de alrededor de tres millones de nóminas cada mes). Además, también incluimos en nuestro análisis a los individuos que reciben algún tipo de transferencia pública relacionada con su participación en el mercado laboral (como un subsidio de desempleo o por ERTE ${ }^{1}$ ). Esta información es imprescindible para poder valorar el impacto de la crisis económica y el efecto que tienen las transferencias públicas.

Así, nuestra muestra comprende todos los ingresos recibidos por los asalariados como nómina cada mes. Las nóminas están identificadas precisamente como un tipo de transferencia bancaria específica, distinta de otros movimientos bancarios, y se incluyen todos los movimientos identificados como nómina agregados

\footnotetext{
${ }^{1}$ Expediente de Regulación Temporal de Empleo.
} 


\section{FIGURA 1}

DISTRIBUCIÓN DE SALARIOS MENSUALES NETOS EN ESPAÑA (Frecuencia en \%)

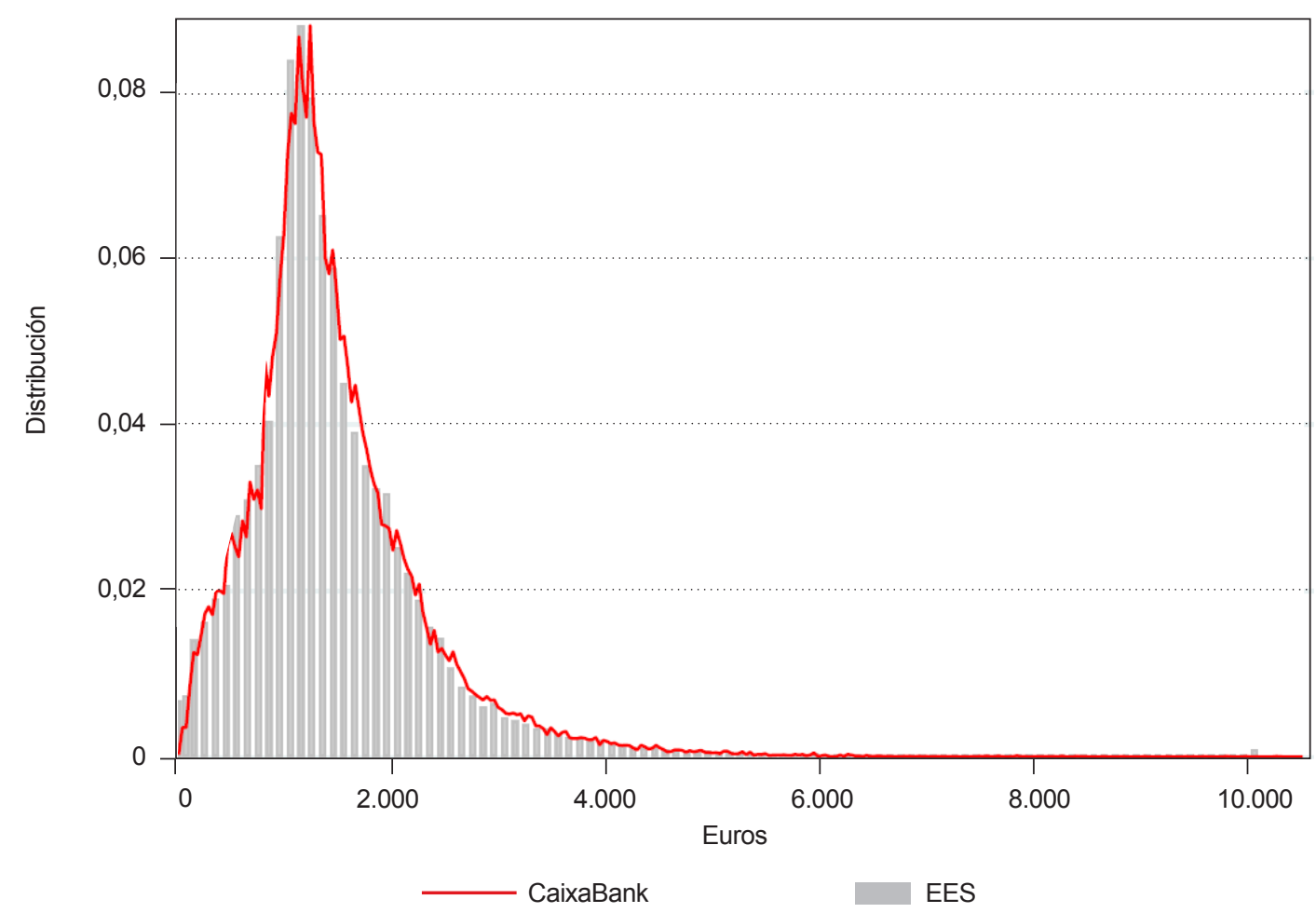

NOTA: Para facilitar la comparación entre muestras, ajustamos la distribución salarial de la EES de 2018 por el aumento salarial promedio entre 2018 y 2019.

FUENTE: CaixaBank Research, a partir de datos internos de CaixaBank y de la Encuesta de Estructura Salarial (INE).

a nivel mensual, independientemente de su cuantía o de la frecuencia en que se recibe (semanal, mensual, etc. $)^{2}$. Las transferencias públicas por ERTE o por desempleo también se identifican de manera precisa al corresponder a un tipo de transferencia específica que realiza la Seguridad Social a los trabajadores que se encuentran en ERTE o desempleo. Al igual que con las nóminas, las transferencias públicas recibidas también se agregan a nivel mensual. Aquellos trabajadores que

\footnotetext{
2 La muestra no incluye a los autónomos, que suelen tener múltiples cuentas bancarias tanto para su uso personal como profesional, por lo que sus ingresos laborales son más difíciles de cuantificar.
}

pierden su trabajo continúan en la muestra, independientemente de si cobran una prestación al estar en ERTE o desempleo o de si no perciben ninguna prestación (en cuyo caso les corresponden cero ingresos). Además, el análisis lo podemos llevar a cabo tanto para el conjunto de la población como para distintos grupos, ya sea en función de la edad, el género u otras características, como la geográfica.

\section{Representatividad de los datos}

La amplia base de datos utilizada es representativa del conjunto de la población, lo que permite valorar el 
TABLA 1

COMPARATIVA DE LOS RATIOS DE PERCENTILES DE LA DISTRIBUCIÓN DE SALARIOS NETOS Y DE LA DISTRIBUCIÓN POR EDAD Y GÉNERO

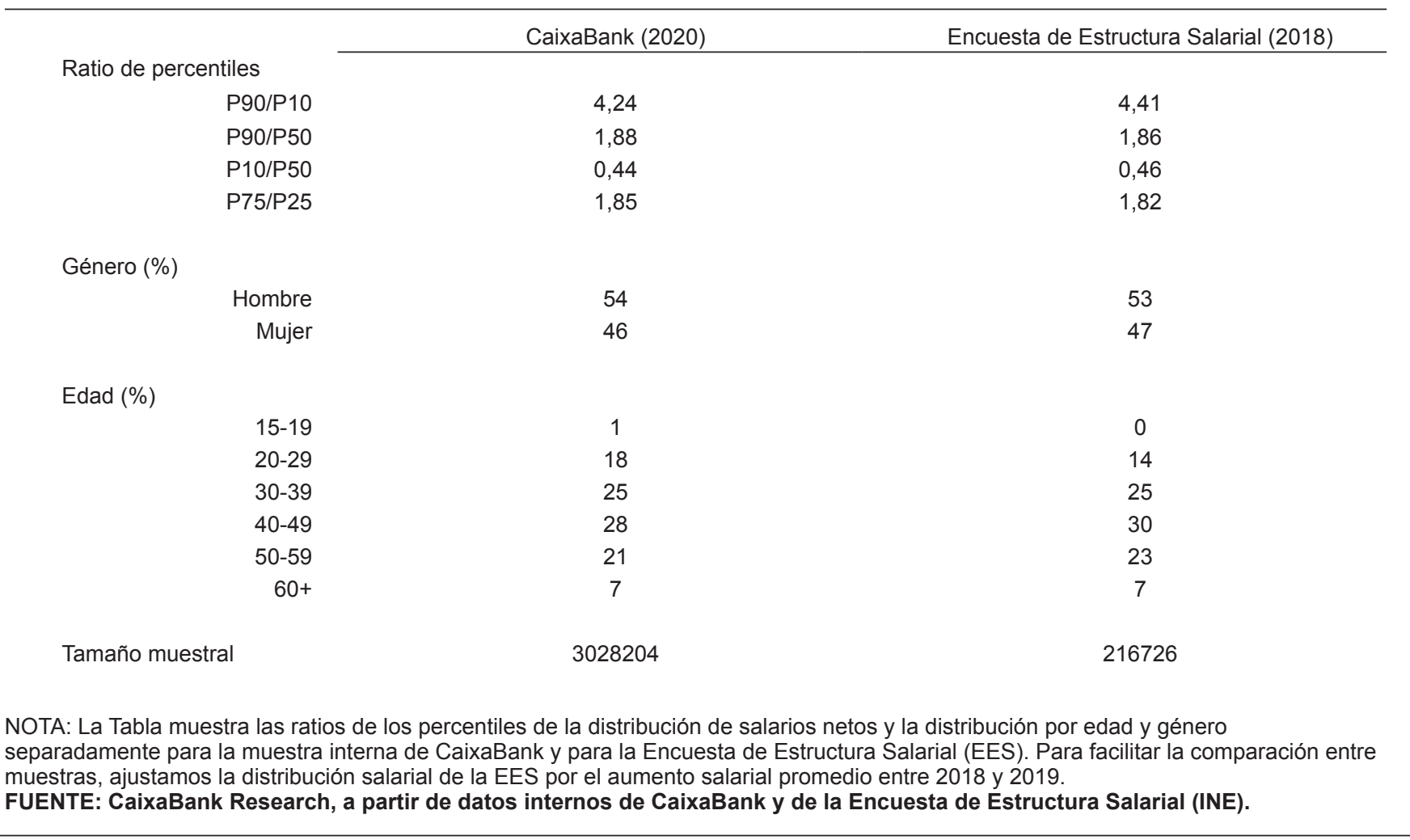

impacto de la crisis sobre los distintos colectivos y la efectividad de las transferencias públicas sobre cada uno de ellos. Para confirmar la representatividad de los datos internos de CaixaBank con datos externos, restringimos la muestra a los asalariados para poder comparar la distribución de las nóminas con los datos de la Encuesta de Estructura Salarial (EES). Como se puede observar en la Figura 1, las dos distribuciones son extremadamente parecidas.

Para corroborar este hecho, comparamos también los ratios de los percentiles de cada distribución en la Tabla 1. Como se puede apreciar, en ambos casos son muy similares. Asimismo, en la Tabla 1 adjunta comparamos las características de las personas con una nómina en CaixaBank con los de la EES. Así, podemos comprobar que el peso de distintos colectivos en los datos de CaixaBank es muy parecido al de la EES y, por tanto, igual de representativo del conjunto de la población asalariada (aunque con un mayor tamaño muestral).

Tener unos datos que sean representativos del conjunto de la población nos permite construir indicadores de desigualdad, como el índice de Gini o las Curvas de Lorenz, para el conjunto de la población y por los distintos subgrupos de población, y analizar su evolución a lo largo del tiempo. Para ello, hemos creado el portal web https://inequality-tracker.caixabankresearch. $\mathrm{com} /$ donde vamos actualizando cada mes las principales métricas, y ponemos a disposición del público los datos a nivel agregado para quien quiera llevar a cabo un análisis con más profundidad. 
4. La desigualdad en España: comparativa del impacto de la crisis financiera y de la crisis provocada por la COVID-19

La recuperación de la actividad, finalmente, es una realidad palpable y que reflejan con claridad los principales indicadores económicos. EI PIB, tras llegar a situarse más de un $20 \%$ por debajo del nivel prepandemia, en el 2T 2021 ya prácticamente había recuperado dos terceras partes del terreno perdido. Pero ¿la recuperación económica está llegando a todos los bolsillos?

La experiencia vivida durante la crisis inmobiliaria y financiera no invita al optimismo. Entre los años 2008 y 2013 el PIB llegó a retroceder más de un 8 \%, y ello conllevó un fuerte aumento de la desigualdad. Lo que todavía fue más preocupante es que la recuperación que se inició a partir de 2014 tardó en hacer mella en todos los hogares. Los indicadores de desigualdad se redujeron muy lentamente durante los años posteriores a la crisis y, de hecho, cuando el pasado año estalló la pandemia, el índice de Gini, el indicador de desigualdad de referencia, todavía se encontraba por encima del nivel de 2008. Esta afirmación es válida tanto para el índice de Gini que mide la desigualdad de renta antes de tener en cuenta las transferencias del sector público (Gini-pre), como para el índice de Gini que analiza la evolución de la desigualdad de renta teniendo en cuenta las transferencias que realiza el sector público (Gini-post).

La crisis económica generada por la pandemia también ha conllevado un fuerte aumento de la desigualdad. El índice de Gini-pre aumentó más de 10 puntos entre los meses de febrero y abril del pasado año ${ }^{3}$. Un aumento histórico, aunque merece la pena resaltar que el aumento de la desigualdad en relación con la

\footnotetext{
3 Índice de Gini elaborado a partir de la información de más de tres millones de nóminas domiciliadas en CaixaBank. Para más información de cómo se construyen los indicadores de desigualdad en tiempo real, a partir de los datos internos de CaixaBank, véase el Informe Mensual de noviembre de 2020 .
}

caída de la actividad fue sustancialmente inferior a la que se produjo durante la pasada crisis inmobiliaria y financiera. Concretamente, en la crisis de 2008-2013, por cada punto porcentual (p. p.) que retrocedió el PIB, el índice de Gini-pre aumentó 0,9 p. p. (Figura 2A). En cambio, en la crisis actual esta sensibilidad se ha reducido a la mitad: por cada punto de PIB, el índice de Gini-pre ha variado 0,4 puntos. El aumento del índice de Gini-post, respecto a la caída del PIB, también se ha reducido a la mitad (Figura $2 \mathrm{~B}$ ). Pero, más allá del cambio en la sensibilidad de los índices de Gini respecto al PIB, es muy destacable que a partir del momento en que la economía se ha ido recuperando, la desigualdad ha empezado a reducirse sin dilaciones.

Los factores de fondo que explican la distinta evolución de la desigualdad en la crisis actual hay que buscarlos en el mercado laboral. Y es que la evolución del empleo explica alrededor del $90 \%$ de la variación del índice de Gini en la actual crisis ${ }^{4}$. Sin lugar a duda, la naturaleza de la actual crisis, muy distinta a la de 2008-2013, es un factor determinante. La recesión anterior fue mucho más larga, y afectó de forma especial a unos sectores concretos, como el inmobiliario, lo que obligó a muchas personas a reorientar su carrera profesional y, por consiguiente, dificultó su reinserción en el mercado laboral. En cambio, la crisis generada por la pandemia está yendo a una velocidad muy superior, y las exigencias de recolocación sectoriales son inferiores. Además, en la actual crisis muchas personas que han dejado de trabajar han contado con un instrumento que les ha permitido mantener la relación laboral con la empresa en la que trabajaban y, cuando la economía se ha reactivado, ello ha facilitado enormemente su rápida reinserción al trabajo: los ERTE.

Finalmente, también cabe destacar que la mejora de los índices de Gini está siendo generalizada entre los

\footnotetext{
${ }^{4}$ Véase Aspachs et al. (2021b) para una explicación detallada de cómo se ha estimado. En la anterior crisis la evolución del empleo explicó alrededor del $80 \%$ de la variación del Gini-pre, según estimaciones de la OECD (2015).
} 

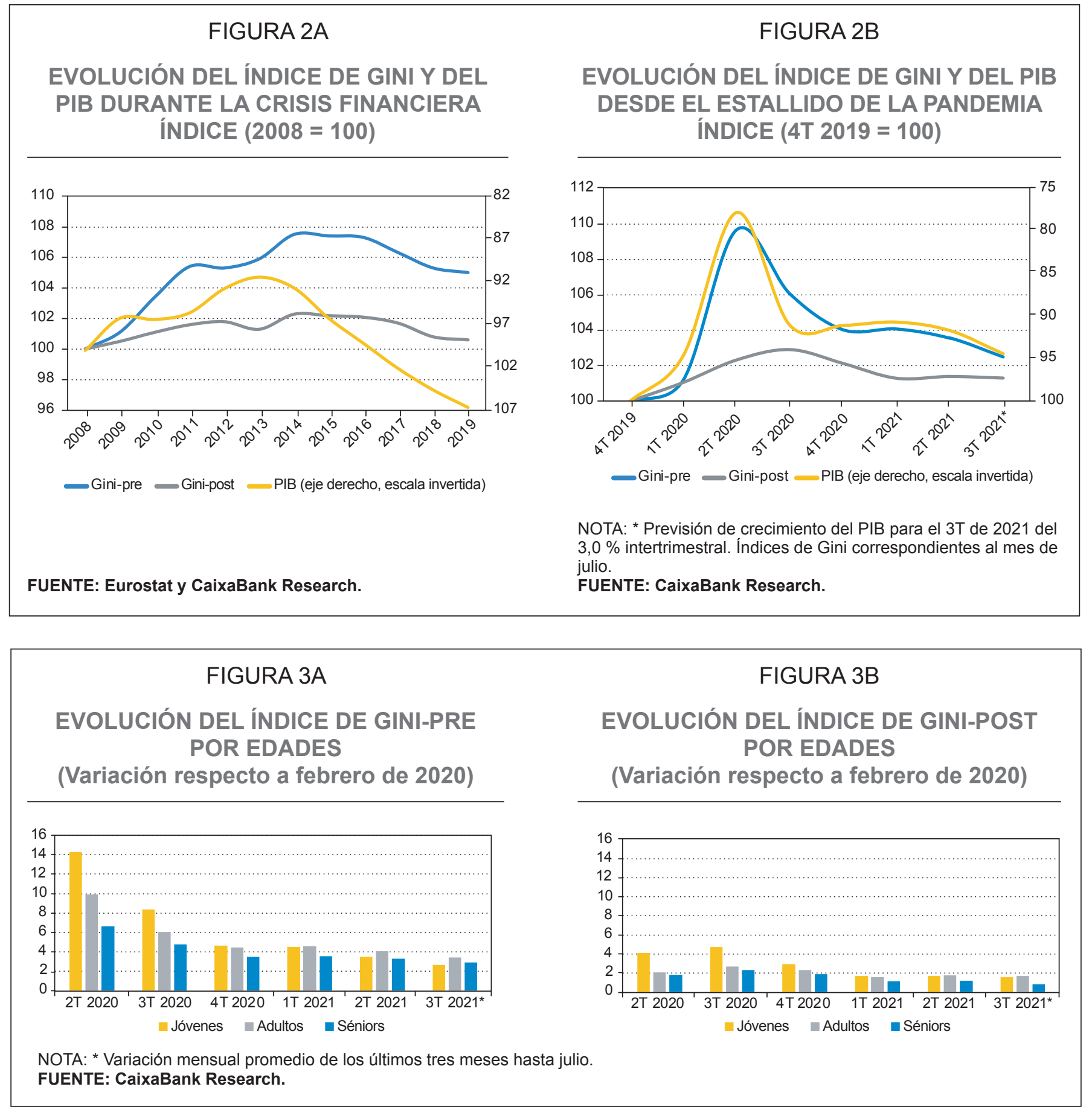

distintos colectivos. Los índices de Gini de los jóvenes y de las personas nacidas fuera de España aumentaron mucho más que en el resto de la población durante los primeros compases de la pandemia. Sin embargo, desde que la actividad económica empezó a reactivarse, la situación de estos colectivos ha mejorado de forma sustancial. En julio ya no se observaban diferencias tan destacables en la variación del índice de Gini por edades (Figuras 3A y 3B). Para el caso de los inmigrantes, los índices de Gini también han mejorado de forma 


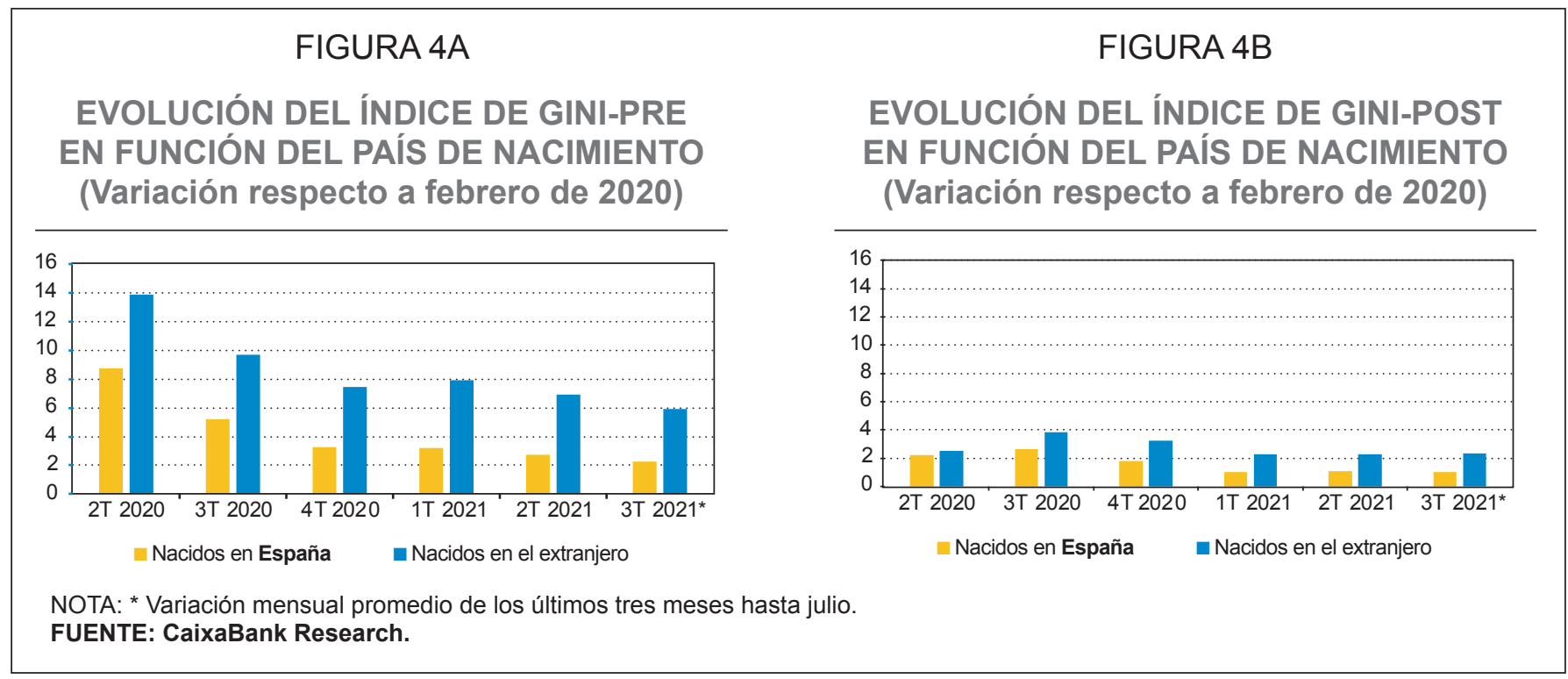

notable, aunque se mantienen algo por encima de los españoles (Figuras $4 \mathrm{~A}$ y $4 \mathrm{~B}$ ).

La evolución más reciente de la desigualdad invita al optimismo, tanto por lo que observamos a nivel agregado, como entre los distintos colectivos. Pero el aumento de la desigualdad respecto al nivel previo a la pandemia sigue siendo destacable. El incremento que registraba el índice de Gini-post en julio de este año, de 1,3 puntos respecto al nivel prepandemia, no se aleja mucho del que se llegó a producir durante la recesión de 2008-2013, de 2,3 puntos. El camino que todavía queda por recorrer hasta alcanzar los niveles previos a la pandemia es amplio. Los próximos trimestres, en los que esperamos que se consolide la recuperación, serán claves para determinar si al final se repite la historia, y la actividad y la desigualdad vuelven a ir por caminos separados. $\mathrm{O}$, por el contrario, esta vez es diferente.

\section{Factores que explican la distinta presión} sobre la desigualdad a nivel regional

La crisis económica generada por la COVID-19 está siendo fortísima, esto es bien conocido, pero también está siendo muy distinta entre regiones. Ello hace que la presión sobre la desigualdad también sea muy heterogénea. Hay varios factores que pueden explicar las grandes diferencias regionales, como la distinta dureza de las medidas aplicadas en cada zona o la distinta estructura productiva. A continuación, analizamos con detalle el papel que han jugado estos factores en la evolución de la desigualdad de cada comunidad autónoma (CC AA). También analizamos más abajo la capacidad del sector público para amortiguar las diferencias regionales. Como verá, ha jugado un papel fundamental, y a pesar de la distinta intensidad del shock en cada CC AA, el aumento de la desigualdad que finalmente se ha producido ha sido muy similar en todas ellas.

Realizamos la siguiente regresión lineal a nivel regional:

$$
\begin{aligned}
& \operatorname{Ln}\left(\left(\operatorname{Gini}_{i t}-\operatorname{Gini}_{i, \text { February2020 }}\right)-\left(\operatorname{Gini}_{i t-12}-\operatorname{Gini}_{i, \text { February2019 }}\right)\right) \\
& =\beta_{o}+\sum_{j=1}^{J} \beta_{j} X_{i j t}+\varepsilon_{i t}
\end{aligned}
$$

Donde Ln ((Gini ${ }_{i t}-$ Gini $\left._{i, \text { February2020 }}\right)$ - (Gini ${ }_{i t-12}$ - Gini $\left.{ }_{i, \text { February2019 }}\right)$ corresponde a la variación en la desigualdad mes a mes en cada región corregida por la variación experimentada en el mismo mes del año anterior (diferencias-en-diferencias). Las variables explicativas $X_{i j t}$ corresponden a las restricciones de 
movilidad en cada CC AA, la composición sectorial de la región, el porcentaje de población urbana, así como una variable indicativa del mes. Las restricciones de movilidad se definen como el porcentaje de movilidad en cada región con respecto al periodo de referencia (14 al 20 de febrero) utilizando datos anonimizados de uso y localización del teléfono móvil de uno de los tres principales operadores de España ${ }^{5}$. La composición sectorial de cada región se define como el porcentaje del PIB en cada uno de los siguientes sectores: Agricultura - sector A de la clasificación NACE (Nomenclatura estadística de actividades económicas de la Comunidad Europea)-, Minería, Energía y Suministro de agua $(B, D, E)$, Industria manufacturera $(C)$, Construcción $(F)$, Comercio minorista, Transporte y Servicios de hostelería $(G, H, I)$, Actividades financieras y de seguros $(K)$, Otros servicios $(J, L, M, N, R, S)$ y Sector público $(O, P, Q)$ (categoría omitida) ${ }^{6}$. El porcentaje de población urbana se calcula utilizando información sobre el municipio del titular de la cuenta bancaria, que se define como urbana si su población es mayor de 30.000 habitantes y la densidad de población es superior a 100 habitantes por kilómetro cuadrado.

La presión sobre la desigualdad salarial ha sido muy distinta en las CC AA. En abril de 2020, cuando la crisis fue más intensa, todas las comunidades autónomas experimentaron un aumento súbito y muy pronunciado de la desigualdad, pero de distinta intensidad. El índice de Gini-pre, que mide la desigualdad salarial sin tener en cuenta las transferencias públicas, aumentó entre 7 y 11 puntos en la mayoría de CC AA. Sin embargo, en algunas, como las Illes Balears y las islas Canarias, el aumento fue muy superior, con variaciones del índice de Gini-pre de 19 y 17 puntos respectivamente. El impacto fue disminuyendo a medida que se reanudó la actividad, y en

\footnotetext{
${ }^{5}$ La movilidad está definida con datos del Ministerio de Transportes, Movilidad y Agenda Urbana, concretamente datos de movilidad urbana e interurbana.

${ }^{6}$ Instituto Nacional de Estadística de España, Cuentas Regionales de España (año 2019).
}

julio de 2021 el aumento respecto a los niveles prepandemia ya «solo» era de 1 a 3 puntos en la mayoría de CC AA. En las Illes Balears e islas Canarias las cifras seguían siendo claramente superiores.

La distinta evolución de la desigualdad salarial en cada región se debe, sobre todo, a la distinta estructura productiva de cada CC AA, ya que el impacto de las medidas que se tomaron fue muy diferente entre sectores. Concretamente, los sectores más afectados fueron el comercio minorista y la hostelería, y también la construcción. Así, el índice de Gini-pre aumentó mucho más en aquellas CC AA en las que estas actividades tienen un mayor peso. Por el contrario, el aumento de la desigualdad fue inferior en las regiones con un mayor peso de sectores económicos que pudieron continuar con cierta normalidad la actividad, como el sector público, el financiero o el de suministros (agua, electricidad, etc.) (Figura 5).

En la Figura 6 trasladamos este análisis a cifras concretas comparativas con el impacto de la composición sectorial para el conjunto de España. Como podemos ver, la estructura productiva de las Illes Balears o las islas Canarias, muy centrada en el turismo, está correlacionada con un aumento del índice de Gini-pre 6,5 y 4,3 puntos mayor, respectivamente, que en el conjunto de España. En el lado opuesto, la Comunidad de Navarra se vio favorecida por su composición sectorial. La industria manufacturera, que tiene un elevado peso en esta Comunidad, ha estado menos afectada por las restricciones, y ello ha amortiguado el aumento del índice de Ginipre en 1,9 puntos en esta región. La Comunidad de Madrid también se ha visto favorecida por su composición sectorial. Esta presenta un elevado peso de los servicios que han podido seguir con relativa normalidad su actividad gracias al teletrabajo, lo que ha amortiguado el aumento del índice de Gini-pre en 1,6 puntos. Cataluña se encuentra en un punto intermedio. Si bien el peso del turismo es relativamente elevado, ello ha sido compensado a nivel agregado por el buen comportamiento, en términos relativos, 


\section{FIGURA 5}

\section{IMPACTO DEL PESO DE CADA SECTOR EN LA VARIACIÓN EN LA DESIGUALDAD SALARIAL EN CADA CC AA DEBIDO A LA COVID-19* (Variación en el índice de Gini por cada punto porcentual de peso)}

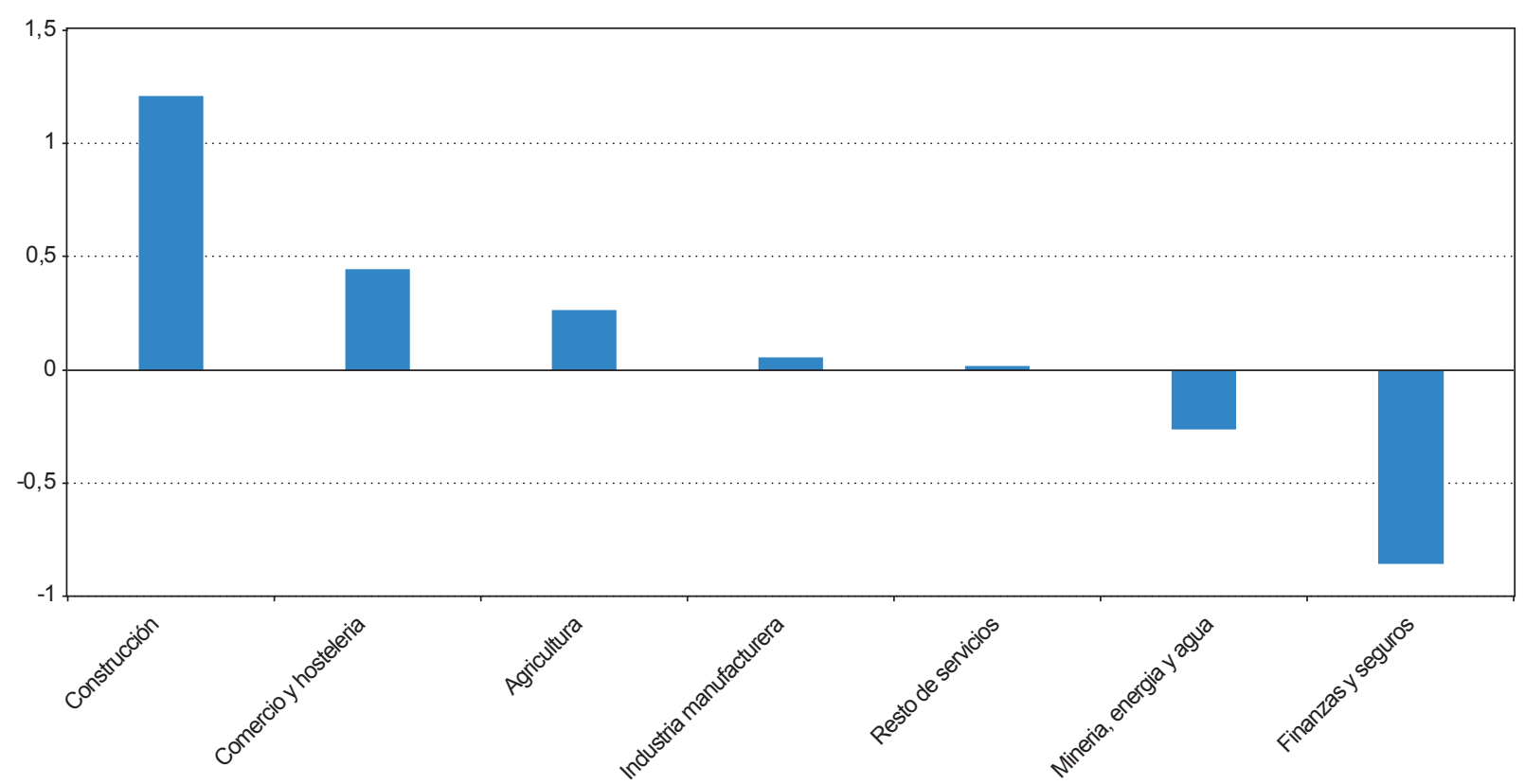

NOTA: * Relación entre cada punto porcentual de peso del sector en la variación de la desigualdad salarial medida por el índice de Gini antes de las transferencias públicas. El sector económico de referencia (impacto cero) es el sector público (incluye sanidad y educación). Impacto promedio estimado con una regresión lineal que controla por las restricciones de movilidad, el porcentaje de población urbana y el mes.

FUENTE: CaixaBank Research, a partir de datos internos.

de la industria, que en esta región también tiene un peso alto. En conjunto, la composición sectorial de Cataluña ha compensado el aumento del índice de Gini-pre en 0,6 puntos.

Las restricciones a la movilidad redujeron drásticamente los desplazamientos en todas las comunidades. En mayo de 2020, la movilidad en el conjunto de España fue un $51 \%$ inferior a la del periodo de referencia pre-COVID, y se detectan ciertas diferencias regionales. Mientras en Madrid la reducción de la movilidad se acercó al $68 \%$, en Andalucía o Galicia el descenso se quedó en el $52 \%$ y $44 \%$, respectivamente. La movilidad se fue recuperando poco a poco en los meses posteriores en todas las CC AA, y en el mes de setiembre de 2021 ya solo se encontraba alrededor de un $5 \%$ por debajo del periodo pre-COVID. Pero durante los meses más duros de la pandemia esta menor movilidad se tradujo en una reducción de la actividad económica, especialmente para aquellos empleados que no podían teletrabajar, lo que impactó en los ingresos salariales de una manera fuerte.

Pongamos cifras a la relación entre la reducción de la movilidad y la desigualdad salarial. En el mes de mayo del pasado año, cuando la caída de la movilidad fue muy severa, ello contribuyó a un aumento del índice de Gini-pre de 2,2 puntos en el conjunto de España. 


\section{FIGURA 6}

\section{VARIACIÓN DE LA DESIGUALDAD RESPECTO AL CONJUNTO DE ESPAÑA: IMPACTO DE LA ESTRUCTURA PRODUCTIVA DE CADA CC AA (Variación del índice de Gini-pre respecto al nivel prepandemia)}

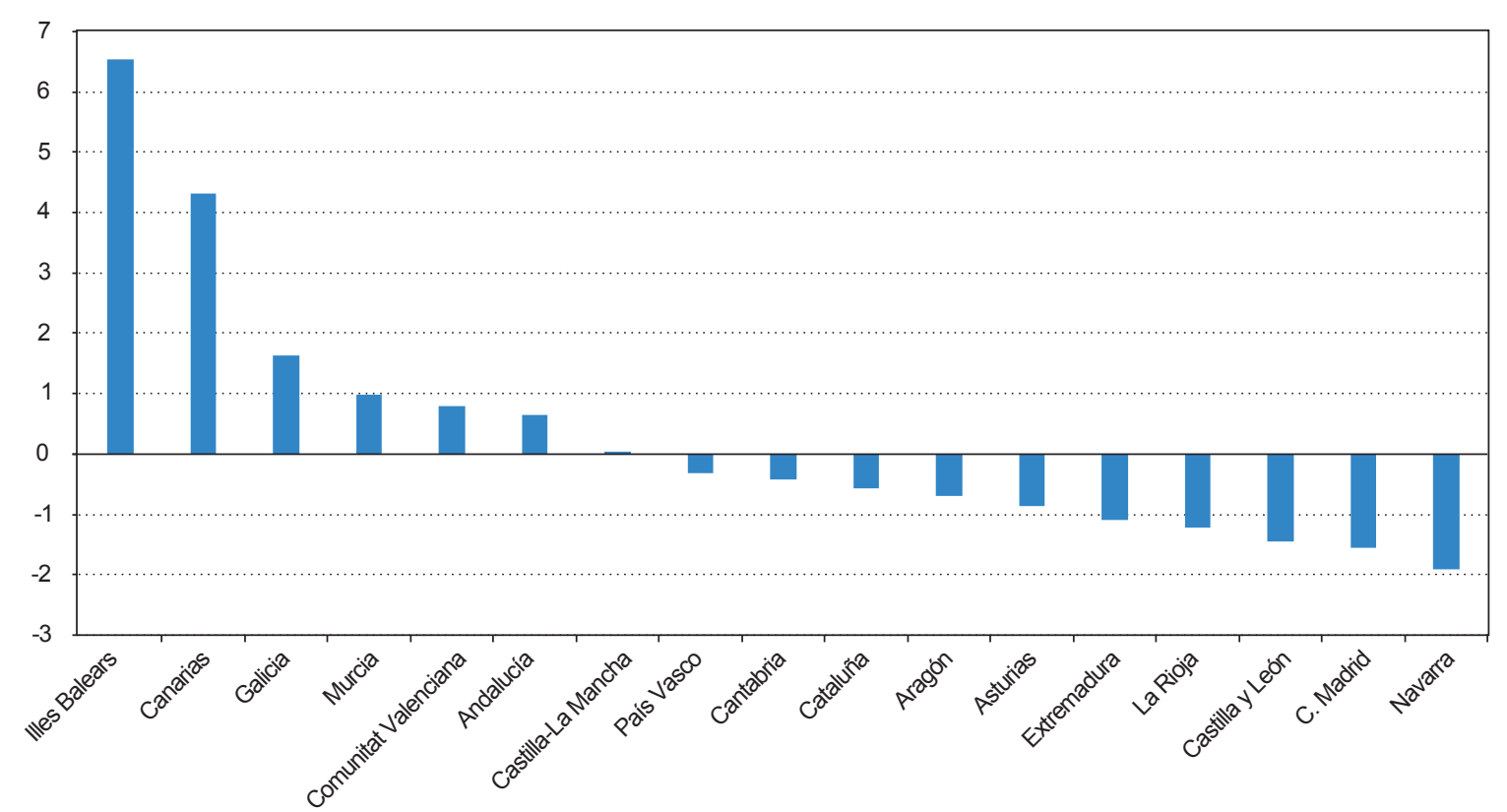

NOTA: * Impacto de la composición sectorial de la CC AA en p.p. de la variación de la desigualdad salarial medida por el índice de Gini antes de las transferencias públicas (en términos comparativos con la composición sectorial promedio de España). Impacto promedio estimado con una regresión lineal que controla por las restricciones de movilidad, el porcentaje de población urbana, así como el mes. FUENTE: CaixaBank Research, a partir de datos internos.

El impacto, por tanto, fue de primer orden y generalizado en todas las CC AA. Dos resultados adicionales a tener en cuenta. Primero, en los meses siguientes, la relación entre la caída de la movilidad y la de la actividad económica se debilitó. O, dicho de otra forma, observamos que poco a poco fuimos capaces de adaptarnos al nuevo entorno para reanudar la actividad económica, con niveles de movilidad inferiores. Ello también se tradujo en menores impactos en la desigualdad salarial. Segundo, las diferencias en la caída de la movilidad que observamos entre regiones explican una parte más pequeña que las diferencias en la estructura productiva, pero no es negligible
(Figura 7). Por ejemplo, en la Comunidad de Madrid, la mayor caída de la movilidad respecto la que experimentó el conjunto de España en el mes de mayo, presionó el índice de Gini-pre al alza en 0,8 puntos más que en España. En cambio, en Galicia, la menor caída de la movilidad amortiguó la caída del índice de Ginipre en 0,3 puntos.

Finalmente, merece la pena destacar que, a pesar de que la intensidad del shock ha sido distinta en cada CC AA, cuando analizamos la evolución del índice de Gini teniendo en cuenta el efecto redistributivo de las prestaciones de paro y las transferencias por ERTE, el índice de Gini-post, las diferencias regionales 


\section{FIGURA 7}

\section{VARIACIÓN DE LA DESIGUALDAD RESPECTO AL CONJUNTO DE ESPAÑA: IMPACTO DE LA MOVILIDAD EN CADA CC AA* (Variación del índice de Gini-pre respecto al promedio de España)}

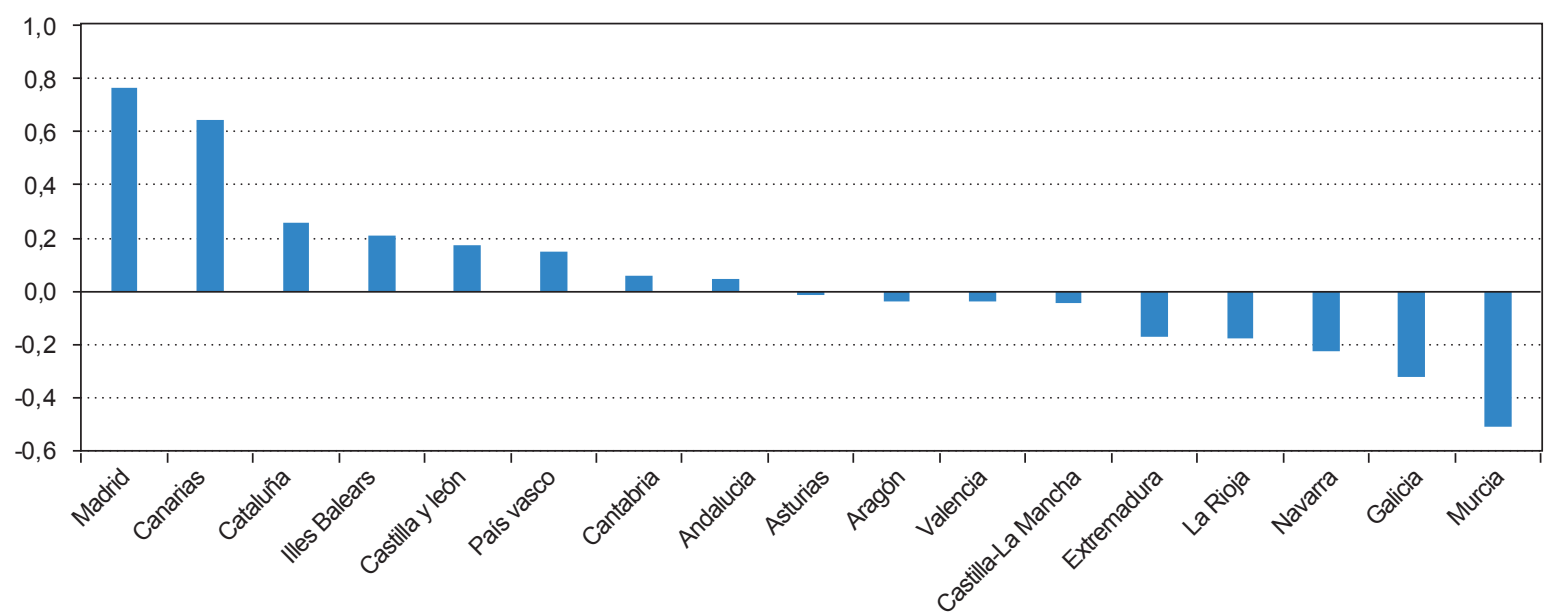

NOTA: * Impacto de la movilidad en p.p. de la variación de la desigualdad salarial medida por el índice de Gini antes de las transferencias públicas (en términos comparativos con el promedio de España). Impacto promedio o estimado con una regresión lineal que controla por las restricciones de movilidad y la composición sectorial.

FUENTE: CaixaBank Research, a partir de datos internos.

prácticamente desaparecen. Concretamente, el $90 \%$ de las CC AA presentan un aumento promedio del índice de Gini-post desde el estallido de la pandemia de entre 1,1 y 2,1 puntos. Dicho de otra forma, con un rango de un punto de diferencia en el aumento del índice encontramos a prácticamente todas las CC AA. En cambio, la dispersión en el aumento promedio del índice de Ginipre es muy superior: con una diferencia de un punto, por ejemplo, un aumento entre 3,8 y 4,8 puntos, solo encontramos al $50 \%$ de las CC AA. El estado del bienestar ha conseguido amortiguar de forma muy destacable la presión del shock económico sobre la desigualdad y, además, prácticamente ha eliminado las diferencias entre regiones. En el siguiente apartado analizamos más a fondo el importante papel que ha jugado el estado del bienestar, y el impacto que podría tener una mejora de la eficiencia con el que se gestiona.
6. La eficacia y la eficiencia del estado del bienestar en la reducción de la desigualdad

A pesar de que la tormenta provocada por la COVID-19 todavía no ha terminado, ya podemos analizar el impacto de la respuesta de las Administraciones públicas (AA PP) en la desigualdad salarial. Las transferencias públicas a los trabajadores en ERTE o desempleados amortiguaron la caída de los ingresos de muchos trabajadores, pero ¿hasta qué punto amortiguaron el aumento de la desigualdad? Respondemos esta pregunta a continuación con los datos internos de CaixaBank, y analizamos también las implicaciones de una mayor eficiencia en la gestión de estas prestaciones.

La actuación de las AA PP jugó un papel importantísimo en esta crisis y mitigó la mayor parte del 
incremento de la desigualdad. En los meses más duros de la pandemia, abril y mayo del pasado año, las prestaciones de paro y, sobre todo, las transferencias realizadas a trabajadores que estaban en ERTE amortiguaron el $80 \%$ del incremento de la desigualdad salarial ${ }^{7}$. Durante los últimos meses, la crisis generada por la pandemia se ha moderado, pero, aun así, entre abril y julio de este año, las transferencias públicas aun compensaban alrededor del $56 \%$ del aumento de la desigualdad salarial.

Todo ello ha supuesto un enorme esfuerzo para la Administración pública. No solo en términos monetarios. El incremento masivo de solicitudes de prestaciones ha sido muy difícil de gestionar, y en muchos casos se han alargado los tiempos de tramitación de los expedientes (Figura 8) ${ }^{8}$.

Para analizar la eficiencia en la gestión de las transferencias públicas realizamos una regresión lineal a nivel regional de la reducción de la desigualdad debido a las transferencias públicas mes a mes:

$$
\operatorname{Ln}\left(\text { GiniPre }_{i, t}-\text { GiniPost }_{i, t}\right)=\gamma_{0}+\sum_{j=1}^{J} \gamma_{j} x_{i j t}+\eta_{i t}
$$

Donde la variable explicada Ln (Gini Pre ${ }_{i, t}$ - Gini Post $_{i, t}$ ) corresponde a la diferencia entre el índice de Gini antes de considerar las transferencias públicas y después de considerarlo. Las variables explicativas $X_{i j t}$ corresponden al tiempo de procesamiento de una prestación de desempleo, las restricciones de movilidad, el porcentaje de población urbana, la composición sectorial de la región, así como el mes. El tiempo de procesamiento de una prestación de desempleo corresponde al tiempo promedio en días que el SEPE

\footnotetext{
${ }^{7}$ En abril y mayo de 2020 se registraron aumentos del índice de Gini-pre (sin tener en cuenta las transferencias del sector público) de 11 puntos. En cambio, el índice de Gini-post (que tiene en cuenta las transferencias del sector público), aumentó en 2,3 puntos. ${ }^{8}$ En 2019, el tiempo promedio de procesamiento de un subsidio de desempleo en España era de 1,22 días.
}

(Servicio Público de Empleo Estatal) tardó en procesar una prestación de desempleo en cada región en cada mes. El resto de variables se ha definido de la misma manera que en el apartado anterior.

Los resultados muestran que para reducir la desigualdad no solo es necesario un buen sistema de ayudas públicas. La eficiencia con la que se gestiona también es clave. Por ejemplo, si en el mes de noviembre de 2020 la tramitación de los subsidios de desempleo hubiera sido un $5 \%$ más ágil en número de días, el índice de Gini se hubiera reducido en 0,2 puntos (Figura 9 ) .

$\mathrm{O}$, dicho de otra forma, si en Cataluña y Madrid el número de días con el que se tramitaba una prestación de paro hubiera aumentado la mitad entre los meses de octubre y diciembre de 2020, el índice de Gini hubiera aumentado 0,9 puntos menos. Es una cifra elevada, pero cabe tener en cuenta que este efecto se refiere solo al retraso debido al tiempo de procesamiento, puesto que los individuos con derecho a recibir una prestación acaban percibiendo los atrasos. De todas formas, ello ilustra el impacto que puede tener una mayor eficiencia de la Administración pública. Reducir el tiempo de procesamiento de los subsidios de desempleo, por ejemplo, mediante una mayor digitalización de los servicios públicos de empleo, tiene un impacto directo y elevado en el bienestar de las familias.

Un mejor funcionamiento del estado del bienestar también puede tener implicaciones en otros terrenos, como la política. Para muestra un botón: analizamos si la reducción de la desigualdad que propiciaron las transferencias públicas tuvo algún impacto en las elecciones autonómicas de Galicia y el País Vasco de julio de 2020. Para el análisis, realizamos una regresión lineal a nivel municipal de la tasa de participación en las elecciones y del voto al partido mayoritario en la región en función de la diferencia entre el índice de Gini antes de considerar las transferencias públicas y

\footnotetext{
${ }^{9}$ Alternativamente, un aumento del $1 \%$ de los casos resueltos en menos de 15 días reduciría la diferencia de desigualdad antes y después de transferencias en un 9,2\%.
} 
FIGURA 8

\section{TIEMPO PROMEDIO DE TRAMITACIÓN DE UN SUBSIDIO DE PARO POR EL SERVICIO PÚBLICO DE EMPLEO ESTATAL (SEPE)*} (En número de días)

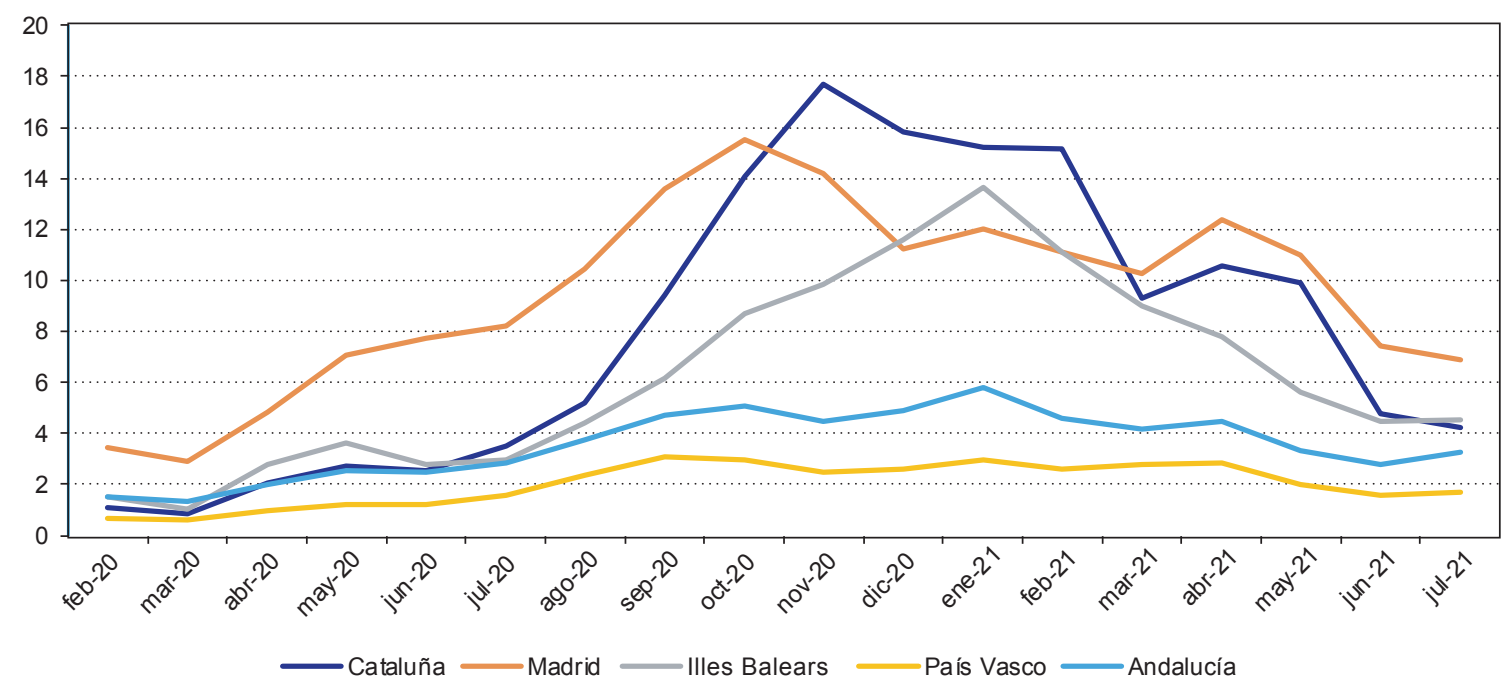

NOTA: * Tiempo promedio de tramitación del procedimiento de prestaciones de desempleo del Servicio Público de Empleo en cada CC AA Media móvil de dos meses del tiempo promedio en cada provincia ponderado por la población.

FUENTE: CaixaBank Research, a partir de datos del SEPE.

después de considerarlo en cada municipio en las dos regiones, y controlando por el tamaño de la población, su distribución por sexo y edad (es decir, proporción de hombres y proporción de personas de 50 a 64 años) y educación (proporción de personas con educación secundaria o más).

Los resultados no dejan lugar a dudas. En aquellas poblaciones en las que el estado del bienestar amortiguó más el aumento de la desigualdad hubo una mayor participación electoral. Concretamente, una reducción de la desigualdad salarial de un punto del índice de Gini aumentó la participación electoral en un $0,3 \%{ }^{10}$. Por otro lado, el voto al partido gobernante estuvo penalizado

\footnotetext{
${ }^{10}$ Para un resultado detallado, así como la especificación completa del
} análisis, ver Aspachs et al. (2021b). en aquellas poblaciones donde fue más necesaria la acción del Estado para reducir la desigualdad. Ambos resultados muestran la relación entre la respuesta pública a la crisis y el voto en las elecciones.

En la mayoría de los países desarrollados pasarán años hasta que no conozcan, de forma fiable, el impacto de la crisis en la desigualdad salarial, y la efectividad de las medidas tomadas por el sector público. En España, en cambio, ya se sabe que la respuesta pública ha sido importantísima, efectiva y que ha tenido implicaciones en múltiples dimensiones. España puede presumir de ser el primer país a nivel mundial de disponer de este tipo de información. $\mathrm{Si}$, además, se incorpora en la toma de decisiones, la efectividad del sector público puede aumentar mucho más. 
FIGURA 9

\section{IMPACTO DEL ESTADO DEL BIENESTAR EN LA REDUCCIÓN DE LA DESIGUALDAD Y MEJORAS EN SU EFICIENCIA* \\ (Variación en el Î́ndice de Gini)}

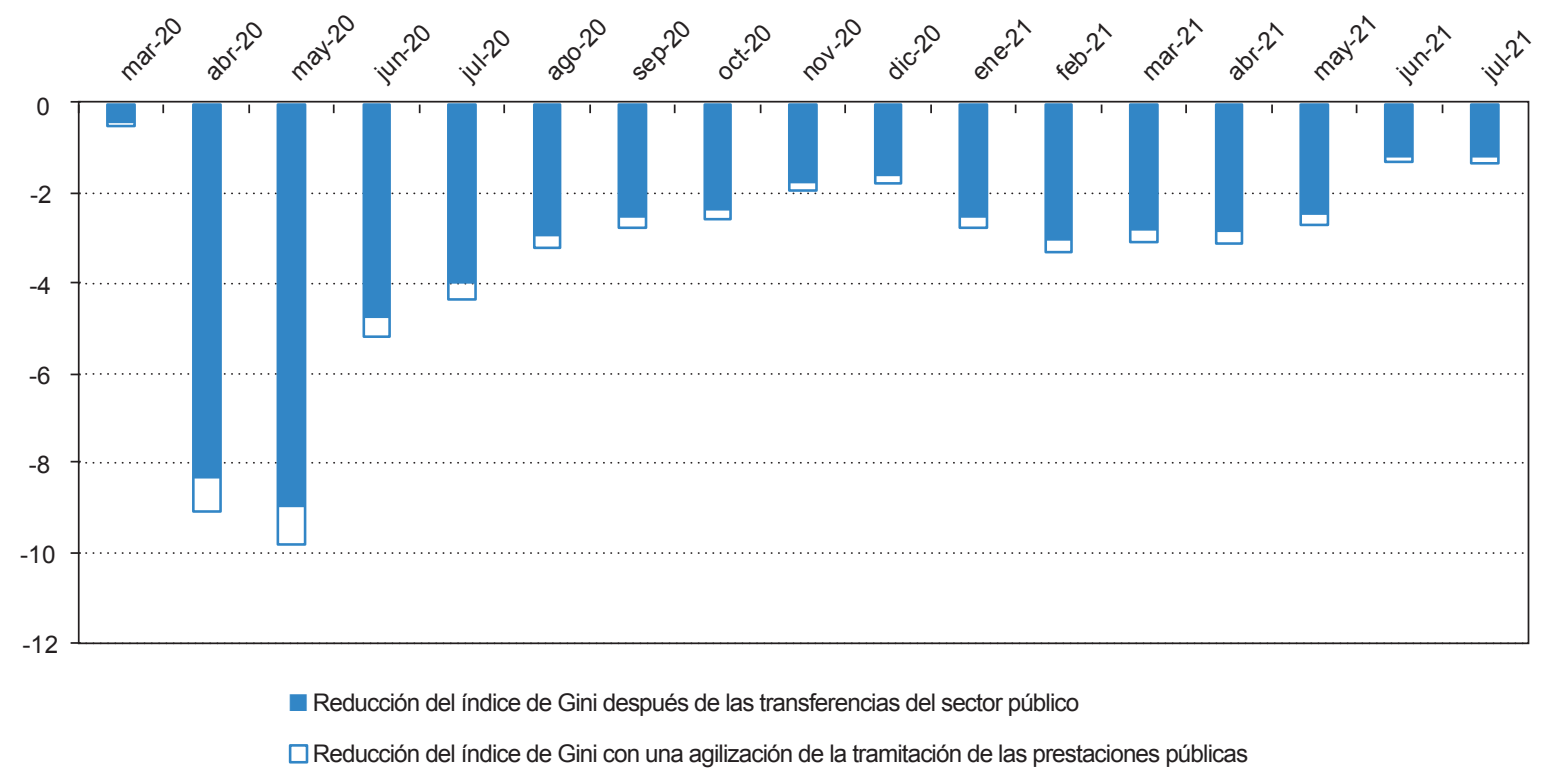

NOTA: * La Figura muestra la diferencia entre el índice de Gini en p.p. después y antes de las transferencias del sector público. Simulación del impacto de una tramitación de los subsidios de desempleo un $5 \%$ más ágil en número de días en la reducción de la desigualdad. Impacto estimado a partir de una regresión de las diferencias entre Gini después y antes de transferencias en función de los días de tramitación de un expediente de desempleo, después de controlar por las restricciones de movilidad, el porcentaje de población urbana, así como el mes.

FUENTE: CaixaBank Research, a partir de datos internos.

7. El empleo, el factor más importante para reducir la desigualdad

A menudo los debates sobre la desigualdad se centran en la dispersión en los niveles salariales y omiten un aspecto muy relevante: la desigualdad de los ingresos salariales depende también de la «intensidad» en el empleo, es decir, de si se está trabajando y con qué regularidad. El caso español es bastante paradigmático, con una alta tasa de temporalidad en el empleo (25,1 \% en el 2 T 2021) y una proliferación de contratos laborales de cada vez menor duración que incide de manera marcada en la desigualdad. Durante la crisis financiera (2007-2011), el fuerte aumento de la desigualdad en España se debió en un 80 \% a la caída del empleo y en un 20 \% a una mayor dispersión de salarios entre trabajadores ${ }^{11}$. Estas proporciones fueron casi las opuestas en el promedio de la OCDE, donde solo el $18 \%$ del aumento en la desigualdad entre 2007 y 2011 se explicó por cambios en el empleo y

\footnotetext{
${ }^{11}$ Cálculos propios a partir de datos de la OECD (2015).
} 
el $82 \%$ fue debido a la mayor dispersión de salarios entre trabajadores. ¿Qué pasó durante la pandemia?

El empleo volvió a ser de nuevo el principal factor detrás de los cambios en la desigualdad salarial durante la crisis de la COVID-19. Por ejemplo, entre febrero y abril de 2020 , casi el $90 \%$ del incremento en la desigualdad salarial (antes de las transferencias públicas) se debió a la caída del empleo, mientras que el resto fue resultado de incrementos en la desigualdad salarial entre trabajadores ${ }^{12}$. Por tanto, para poder incidir en la evolución de la desigualdad, es necesario revisar las dinámicas en el empleo y, en particular, las de los colectivos más afectados.

La probabilidad de continuar empleado no fue igual para todos los trabajadores durante la COVID-19. Al analizar qué ocurrió, durante la pandemia, a aquellas personas que estaban trabajando los meses previos a que esta estallara. Concretamente, a partir de marzo de 2020, cuando se empezaron a tomar medidas para reducir la movilidad y la actividad, la probabilidad de continuar empleado ${ }^{13}$ se redujo de forma notable para el conjunto de la población.

Realizamos una regresión a nivel individual de la probabilidad de estar empleado mes a mes en función de las características individuales (género, edad, país de nacimiento) y el quintil de ingresos del trabajador antes de la pandemia (i.e. febrero 2020), controlando también por las restricciones de movilidad a nivel provincial, así como la provincia:

$$
E_{j i t}=\sum_{k=1}^{5} \alpha_{k t} l\left(q_{k}\right)+\sum_{g=1}^{G} \beta_{g t} X_{i j t g}+\sum_{j=1}^{49} \delta_{j} l(\operatorname{Prov}=j)+\varepsilon_{i j t}
$$

Pero el impacto fue muy distinto en función de las características de cada trabajador (Figura 10). Los trabajadores con menores ingresos salariales antes

\footnotetext{
12 Véase Aspachs et al. (2021b), para una explicación detallada de la estimación.

${ }^{13}$ Para este ejercicio no consideramos a aquellos trabajadores en ERTE como empleados, puesto que no trabajan mientras están en ERTE, y restringimos el análisis de marzo a noviembre de 2020.
}

de la pandemia fueron aquellos con mayor probabilidad de no estar empleados en los meses siguientes. Concretamente, para los trabajadores en el quintil de salarios más bajo, la probabilidad de estar empleado se redujo hasta en un $38 \%$ en mayo de 2020 , una incidencia 23 p.p. superior que para los trabajadores del quintil más alto. Estas diferencias se redujeron algo en los siguientes meses, aunque los trabajadores en los quintiles inferiores de salarios siguieron con una mayor probabilidad de no estar empleados que los de los quintiles superiores.

Por edades, los jóvenes han sido el colectivo más afectado por la pandemia en el mercado laboral. En mayo de 2020 la probabilidad de estar percibiendo una nómina era 18 p.p. menor que para los trabajadores de mayor edad, y la recuperación en los siguientes meses fue solo parcial. La probabilidad de estar empleado también se redujo más para los trabajadores nacidos en el extranjero que para los nacidos en España. El gap en la probabilidad de estar empleado era de 8 p.p. en abril 2020 y sorprendentemente siguió aumentando con los meses, llegando hasta los 11 p.p. en noviembre. Las mujeres tuvieron una menor probabilidad que los hombres de continuar empleadas en los primeros meses de la pandemia, con un gap en la probabilidad de empleo que superó los 4 p.p. y no empezó a recuperarse hasta septiembre.

Los trabajadores con menores ingresos, jóvenes, inmigrantes y de sexo femenino han sido, de nuevo, los más impactados en el mercado laboral. La recuperación del empleo aún no es total, y menos aun para los colectivos más vulnerables. Es por ello que el incremento de la desigualdad respecto al nivel prepandemia es aún considerable y el empleo, como principal factor que afecta a la desigualdad, volverá a ser la principal palanca para corregirlo. Mejorar la empleabilidad de los trabajadores para que nadie se quede atrás, así como aumentar su «intensidad» en el empleo, son las herramientas clave para que el aumento en la desigualdad no se convierta en una cicatriz permanente de la pandemia. 


\section{FIGURA 10}

PROBABILIDAD EN EL EMPLEO DURANTE LA COVID-19*

(Variación en la probabilidad)**

(a) Quintil de ingresos pre-pandemia

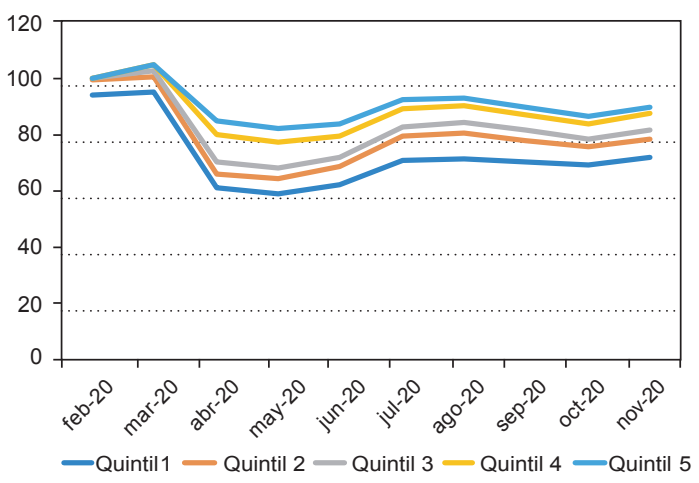

(c) Pais de nacimiento (referencia: España)

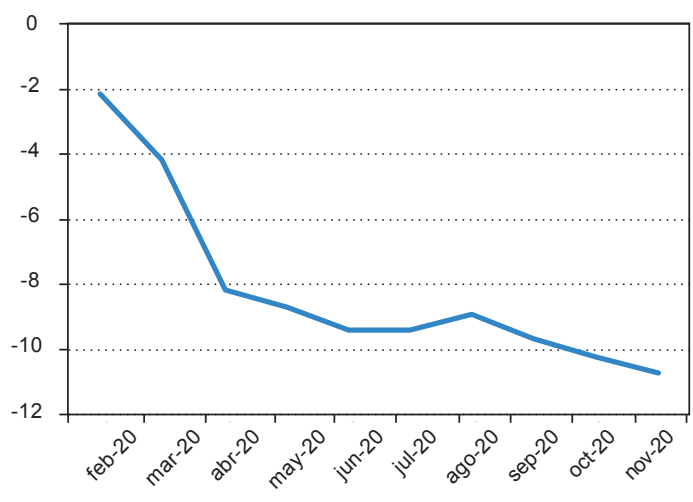

- Inmigrante (b) Edad (grupo de referencia: trabajadores de 55 a 65 años)

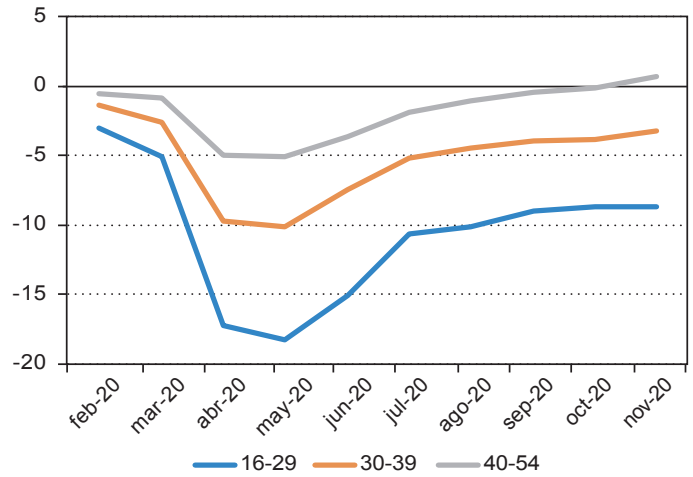

(d) Género (referencia: hombres)

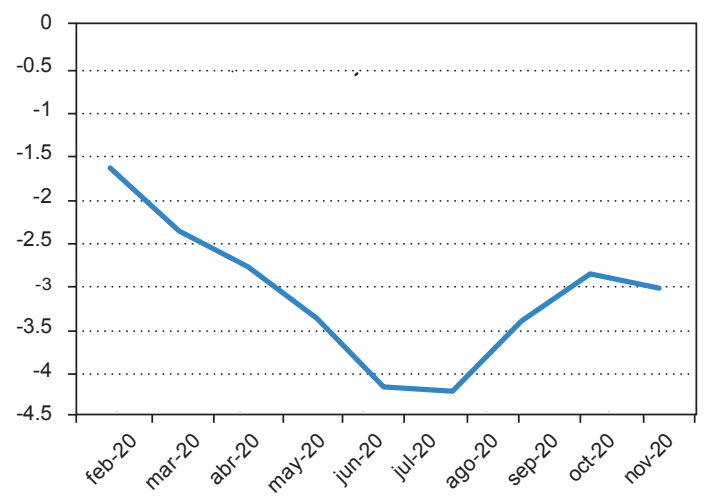

- Mujer

NOTA: * Coeficientes resultado de una regresión a nivel individual de la probabilidad en p.p.de estar empleado mes a mes, controlando por las restricciones de movilidad a nivel provincial, así como la provincia y en función de las características individuales (género, edad, país de nacimiento) y el quintil de ingresos del trabajador antes de la pandemia (i.e. febrero 2020) excepto para el caso de género. ** Para los quintiles de ingresos la Figura muestra la probabilidad de empleo y no la variación.

FUENTE: CaixaBank Research, a partir de datos internos.

\section{Conclusiones}

En definitiva, creemos que las herramientas desarrolladas en este estudio para seguir la evolución de la desigualdad en tiempo real pueden ser una herramienta de gran utilidad. Por un lado, permiten saber la situación salarial de la población durante un periodo de crisis, o de expansión, y esto puede ser útil para detectar a tiempo eventuales desequilibrios o cambios repentinos en la distribución de ingresos. Por otro lado, también es una herramienta que puede ayudar a valorar la efectividad de las políticas puestas en marcha por el sector público, tanto por el conjunto de la población, como por los colectivos más vulnerables. 
En este sentido, hemos podido constatar cómo durante la crisis económica generada por la COVID-19 la desigualdad está experimentando una fuerte volatilidad. En el tramo inicial de la crisis experimentó un aumento muy fuerte, debido a las fuertes restricciones a la movilidad que se implementaron y al impacto que estas tuvieron en algunos sectores.

En España la mejora de los principales indicadores de desigualdad ha sido muy destacada desde que la actividad económica se empezó a recuperar. De todos modos, aún no se han recuperado los niveles previos a la pandemia, y algunos de los colectivos más vulnerables siguen estando especialmente afectados por la crisis generada por la COVID-19. Es importante que la recuperación económica y del mercado laboral se consolide los próximos trimestres, y que las políticas públicas sigan teniendo un rol fundamental para que todas las personas se puedan terminar beneficiando.

\section{Referencias bibliográficas}

Aspachs, O., Durante, R., Graziano, A., Mestres, J., ReynalQuerol, M. \& Montalvo, J. G. (2021a). Tracking the impact of COVID-19 on economic inequality at high frequency. PloS ONE, 16(3). https://doi.org/10.1371/journal.pone.0249121

Aspachs, O., Durante, R., Graziano, A., Mestres, J., ReynalQuerol, M. \& Montalvo, J. G. (2021b). Real-Time Inequality and the Welfare State in Motion: Evidence from Covid-19 in Spain. Economic Policy, de próxima publicación.

Bick, A. \& Blandin, A. (2021). Real-time labor market estimates during the 2020 coronavirus outbreak. SSRN, No. 3692425.

Chetty, R., Friedman, J., Hendren, N., Stepner, M. \& The Opportunity Insights Team. (2020). The economic impacts of COVID-19: Evidence from a new public database built from private sector data. National Bureau of Economic Research, Working Papers No. 27431.

Cicala, S. (2020, April). Early economic impacts of covid-19 in Europe: A view from the grid. Energy Policy Institute at the University of Chicago, Working Papers.

OECD, Organisation for Economic Co-operation and Development. (2015). In it together. Why less inequality benefits all. OECD Publishing, Paris. https://doi.org/10.1787/9789264235120-en 